\title{
Economic Dynamic Model for Small Islands*
}

\author{
AKIO MATSUMOTO* \\ Faculty of Economics, Chuo University 742-1, Higashi-Nakano, Hachioji Tokyo, 192-0393 Japan
}

(Revised 14 April 2001)

\section{INTRODUCTION}

The study constructs an economic dynamic model for small islands based on the Easter Island study by Brander and Taylor (1998) (BT henceforth). It deals with analytical as well as numerical representations of evolutions of a small island economy, evolutions of which are characterized by a two-fold process of transformation: environmental degradation and population growth. Its main purpose is to investigate under what circumstances, the small island economy can survive and under what other circumstances, it must collapse.

Archeological studies have suggested that many Pacific islands followed similar evolutionary patterns of natural resource and population dynamics; rapid population growth, resource degradation, economic decline and then population collapse. Brander and Taylor's study reconsiders archeological and anthropological evidences from economic point of view. In particular, BT present a general equilibrium model of renewable resource and population dynamics to explain the rise and fall of Easter Island for 1400 years between about the 4th century and the middle of the 18th century. It indicates that an economic model linking resource-dynamics and population-dynamics may explain, not only the sources of past historical evolutions discovered in those small islands but also a possibility of sustainable growth of our world economy in which rapidincreasing population and rapid-degrading environment become a serious problem. And, in fact, the research from the economic perspective for such explanations has already begun; see Erickson and Gowdy (2000), Daltion and Coats (2000) and Pezzy and Anderies (2000).

In the existing literature of Easter Island, however, not much has been revealed with respect to a "history" of Easter Island. It has been believed that a small group of Polynesians arrived at the island around 400 A.D., deforestation occurred around 1000 A.D., most of statures were carved during 1000-1400 A.D., and so forth. Based on this "conventional wisdom", BT as well as other researchers investigated a dynamic pattern of natural resource and populations. However, the "wisdom" is still one of possible hypotheses and not fully confirmed yet. In particular, according to Intoh [2000], recent re-considerations of archeological evidences on the island suggest that the small group settled in the island between 410 and 1270 A.D. $\neq$ This new finding is inconsistent with the traditional wisdom. In other word, we may have a different history of Easter Island, even though the available historical evidences are the same. It is thus imperative to construct a model for small islands that can generate various patterns of dynamics in order to deal with such ambiguous characteristics of archeological evidences.

In this study, we reconstruct BT's continuous-time model of Easter Island in discrete steps for three reasons. First concerns the theoretical reason. It is of sure interest to see whether theoretical results obtained in a continuoustime model are carried over to a discrete-time model as well. Second concerns the numerical reason. Since there are no general results on global dynamics for non-linear structure, numerical simulations are a useful procedure for dynamic characterization. However the simulation analysis requires a discretization of the continuous-time model. Thus constructing a non-linear model in discrete steps is a natural direction to go. Lastly and more importantly, a discretization of a continuous-time model allows us to choose a length of "unit time" that is a matter of fundamental importance for a construction of dynamic model. It further allows for the possibility of rich dynamics ranging from stable dynamics, periodic oscillations and aperiodic behavior involving more extreme or moderate "feast and famine" pattern of cyclical evolutions. Our departure from BT's continuous-

\footnotetext{
*This is a revised version of the manuscript entitled, Rise and Fall of Easter Island, which was presented at the Islands of the World VI conference held in the Isle of the Sky, UK, 16-20 of October, 2000. Annual Meeting of Japan Regional Science held in Sendai, Japan, 3-4 of November, 2000 and the Regional Science Association North American Meeting held in Chicago, US, 9-12 of November, 2000

*Tel.: +81-426-74-3351. Fax: +81-426-74-3425. E-mail: akiom@tamacc.chuo-u.ac.jp
}

${ }_{7}^{\ddagger} 700$ A.D., an average year, is often used for the arrival year in the archeological analysis of Easter Island, but only for the sake of convenience. 
time model seems a minor modification, but our arrival on the main results obtained in this study shows a major difference; the choice of time domain can be critical in achieving the non-sustainable growth (i.e. a pattern of overshoot-and-collapse) or the sustainable growth (i.e. population stability and natural resource conservation).

The paper proceeds as follows. The second section recapitulates BT's model as a basic economic model of small islands. Third section develops a discrete-time version and considers its dynamic characterization. Fourth section makes numerical simulations to complement the analytical considerations in the third section. The fifth section concludes the study.

\section{ECONOMIC MODEL FOR SMALL ISLANDS}

Since our analysis is based on BT's dynamic model of renewable resource and population, we recapitulate their model in this section. See BT's paper for more details.

The model describes the dynamics of an economy with two goods; the harvest of the renewable resource and some other goods called manufactured goods. The model functions as follows. At time $t$, the stock of natural resource $S(t)$ and the size of population $L(t)$ are given. Producers determine their demands for labor and supplies of goods so as to maximize their profits. The manufactured goods are produced with constant returns to scale using only labor while harvesting of the resource is carried out according to the Schaefer harvesting production function,

$$
H^{\mathrm{s}}(t)=\alpha S(t) L_{H}(t)
$$

where $H^{\mathrm{s}}(t)$ is the harvest supplied by the agricultural sector, $L_{H}(t)$ is the labor used in resource harvesting and $\alpha$ is a positive constant. A representative consumer is endowed with one unit of labor and is assumed to have a Cobb-Douglas utility function,

$$
u(h, m)=h^{\beta} m^{1-\beta},
$$

where $h$ and $m$ are individual consumption of the resource good and of manufactures, and $\beta \in(0,1)$. Each consumer supplies one unit of labor and demands both goods so as to maximize his utility. Prices are adjusted to establish temporary equilibrium in each of three markets; harvest market, manufactured good market and labor market. As a result of the specified utility function and production functions, the fixed proportion of the total population is employed in the agricultural section, $L_{H}(t)=\beta L(t)$ and thus the resource harvest is $H(t)=\alpha \beta S(t) L(t)$ at the temporary equilibrium state. After finishing transactions in each market, new values of natural resource and the size of population are determined at the next instant of time. With those new values, the process repeats itself until the temporary equilibrium is attained.
Dynamics of temporary equilibrium is described as follows. The change in the stock at time $t$ is determined by the natural growth rate $G(S(t))$ minus the harvest rate, $H(t)$;

$$
\frac{\mathrm{d} S(t)}{\mathrm{d} t}=G(S(t))-H(t)
$$

The logistic functional form for $G$ is assumed, $G(S(t))=r(1-S(t) / K) S(t)$ where $K$ is the maximum possible size for the resource stock, and $r$ is an intrinsic growth rate of natural resource, and both are positive constants. A change of population depends on a difference between an underlying mortality rate and fertility rate. The base or exogenous mortality rate, denoted as $n$, is assumed to be a positive constant. " Following the formulation of Malthusian population dynamics, BT assume that per capita consumption of the resource good increase fertility and/or decreases mortality. BT let $\phi(H(t) / L(t))$ be a fertility function where $\phi$ is positive constant. In consequence, the population growth rate is

$$
\frac{1}{L(t)} \frac{\mathrm{d} L(t)}{\mathrm{d} t}=\left(-n+\phi \frac{H(t)}{L(t)}\right),
$$

where the first factor on the right-hand side is the exogenous mortality rate and the second is the endogenous fertility rate. Thus substituting the logistic function into the natural growth rate and the optimal harvest, $H(t)=$ $\alpha \beta S(t) L(t)$, into the fertility function yield the dynamic system,

$$
\left\{\begin{array}{l}
\frac{\mathrm{d} S(t)}{\mathrm{d} t}=\left\{r\left(1-\frac{S(t)}{K}\right)-\alpha \beta L(t)\right\} S(t), \\
\frac{\mathrm{d} L}{\mathrm{~d} t}=(-n+\alpha \beta \phi S(t)) L(t) .
\end{array}\right.
$$

This is a two-dimensional dynamic system of differential equations and is a variation of the Lotka-Volterra predator-prey model, in which the human is the "predator" and the resource stock is the "prey". The following is the main result of BT.

THEOREM 1 (Proposition 4 of Brander and Taylor, 1998) When an interior steady state exists, the local behavior of the system is as follows. (i) Steady state $1(L=0, S=0)$ is an unstable saddle point steady state allowing an approach along the $S=0$ axis. (ii) Steady state 2 ( $L=$ $0, S=K$ ) is an unstable saddlepoint steady state allowing an approach along the $L=0$ axis. (iii) Steady State 3 $(L>0, S>0)$ is a stable steady state and a "spiral node" with cyclical convergence if $n r / K \phi \alpha \beta+4(n-$ $K \phi \alpha \beta)<0$ and an "improper node" allowing monotonic convergence if not.

\footnotetext{
"Only for the sake of simplicity, we denote the base rate as $n$ while BT use $d-b$ where $d$ is the mortality rate, $b$ is the fertility rate.
} 


\section{DISCRETE-TIME DYNAMIC MODEL}

\section{Discretisation}

Euler's difference scheme for the continuous system Eq. (1) takes the form,

$$
\left\{\begin{array}{l}
\frac{S(t+\Delta t)-S(t)}{\Delta t}=\left\{r\left(1-\frac{S(t)}{K}\right)-\alpha \beta L(t)\right\} S(t) . \\
\frac{L(t+\Delta t)-L(t)}{\Delta t}=\{-n+\phi \alpha \beta S(t)\} L(t),
\end{array}\right.
$$

where $\Delta t$ denotes time step. As $\Delta t \rightarrow 0$, the discrete system converges to the continuous system. Roughly speaking, a discrete system can give rise the same dynamics as a continuous systems if $\Delta t$ is small. It also has a possibility for generating qualitatively different dynamics if $\Delta t$ is large. In this sense, the discrete system with $\Delta t>0$ generalizes the corresponding continuous system. In the following, we first simplify the discretised system Eq. (2) by changing variables, ${ }^{\S}$ explore what dynamics the simplified model can produce and examine what implications would the values of model's parameters have on the dynamic behavior of the small island economy.

Eq. (2) can be, after arranging terms, written as

$$
\left(S_{t+1}, L_{t+1}\right)=F\left(S_{t}, L_{t}\right): R^{2} \rightarrow R^{2}
$$

where

$$
\left\{\begin{array}{l}
S_{t+1}=\left\{(1+r \Delta t)-\frac{r \Delta t}{K} S_{t}-\alpha \beta \Delta t L_{t}\right\} S_{t} \\
L_{t+1}=\left\{(1-n \Delta t)+\alpha \beta \phi \Delta t S_{t}\right\} L_{t}
\end{array}\right.
$$

The state of the system at time $t,\left(S_{t}, L_{t}\right)$, is mapped to $\left(S_{t+1}, L_{t+1}\right)=F\left(S_{t}, L_{t}\right)$, the state of the system one unit of time afterwards. In order that the dynamics system generates economically feasible solutions, it is required that not only $\left(S_{t}, L_{t}\right) \geq 0$ but also $\left(S_{t+1}, L_{t+1}\right) \geq 0$ which are alternatively put as

$$
\begin{gathered}
(1+r \Delta t)-\frac{r \Delta t}{K} S_{t}-\alpha \beta \Delta t L_{t} \geq 0 \quad \text { and } \\
(1-n \Delta t)+\alpha \beta \phi \Delta t S_{t} \geq 0 .
\end{gathered}
$$

By the second equation of Eq. (3), $L_{t+1}=(1-n \Delta t) L_{t}$ describes the population change when no economic activities take place (i.e. $H_{t}=0$ ). Put it differently,

$$
\frac{L_{t+1}-L_{t}}{L_{t}}=-n \Delta t
$$

which implies $n \Delta t$ is the exogenous mortality rate per the time-step. Here, $n \Delta t \geq 1$ means an extinction of humans in one period as a negative population size is not acceptable. Humans can become extinct if "one period" is so long, in addition to the absence of natural resource. However, in this study, to avoid such an extreme and uninteresting case, we mainly focus on a "moderate case" in which "one period" is less than the inverse of the exogenous instantaneous (or point) growth rate of population (i.e. $\Delta t<1 / n$ ).

Assumption $(1-n \Delta t) \geq 0$.

This assumption makes $L_{t+1} \geq 0$ for all $t$. The remaining conditions, $\left(S_{t}, L_{t}\right) \geq 0$ and $S_{t+1} \geq 0$, are satisfied if the dynamic system Eq. (3) is an into-mapping from the following region $\mathbb{R}$ to itself,

$$
\mathbb{R}=\{(S, L) \mid S \geq 0, L \geq 0, \quad \text { and } \quad g(S) \geq L\},
$$

where

$$
g(S) \equiv \frac{1+r \Delta t}{\alpha \beta \Delta t}-\frac{r}{K \alpha \beta} S
$$

The following theorem clarifies under what parameter configurations the dynamic system Eq. (3) can generate economically feasible solutions.

TheOREM $2 F(\mathbb{R}) \subset \mathbb{R}$ if $0 \leq r \Delta t \leq 3$ and $(n, K \alpha \beta \phi) \in$ $\mathbb{N}$ where $\mathbb{N}$ is defined by

$\mathbb{N}=\left\{(n, K \alpha \beta \phi) \mid 0 \leq n \leq \frac{1}{\Delta t}, 0 \leq K \alpha \beta \phi \leq \frac{r(1+\sqrt{n \Delta t})^{2}}{(1+r \Delta t)}\right\}$

Proof See Appendix A.

\section{Stationary State}

Since Eq. (3) is resemble to a Lotka-Volterra predatorprey model, it is expected that it can generate various dynamics according to various values of parameters; dynamics includes stable stationary points, periodic points and aperiodic points. We will find stationary states of the model and then investigate a possibility of such various dynamics.

Consider the $\Delta S=0$ locus and the $\Delta L=0$ locus where $\Delta S=S_{t+1}-S_{t}$ and $\Delta L=L_{t+1}-L_{t}$. An intersection of those loci determine a stationary state of the discrete-time system. By the first equation of Eq. (3), $\Delta S$ is $\Delta S=\left(r-\frac{r}{K} S_{t}-\alpha \beta L_{t}\right) \Delta t S_{t}$.

\footnotetext{
be written as

$$
\begin{aligned}
& S_{k+1}=\left\{(1+r \Delta t)-\frac{r \Delta t}{K} S_{k}-\alpha \beta \Delta t L_{k}\right\} S_{k}, \\
& L_{k+1}=\left\{(1-n \Delta t)+\alpha \beta \Delta t S_{k}\right\} L_{k} .
\end{aligned}
$$
}

${ }^{\S}$ A variable $x(t)$ in continuous time can be written by $x\left(t_{k}\right)$ in discrete time. Set $t_{k}=\Delta t \cdot k(k=1,2, \ldots)$. Then given $\Delta t>0$, the variable can be expressed as follows; $x\left(t_{k}\right)=x(\Delta t \cdot k)=x_{k}$ and $x\left(t_{k}+\Delta t\right)=x(\Delta t \cdot(k+1))=x_{k+1}$. Thus, by the same token, the discretized dynamic system, Eq. (2) can

Length of one period is equal to $\Delta t$. For notational convenience, replacing $k$ with $t$ yields the following discrete-time system. 

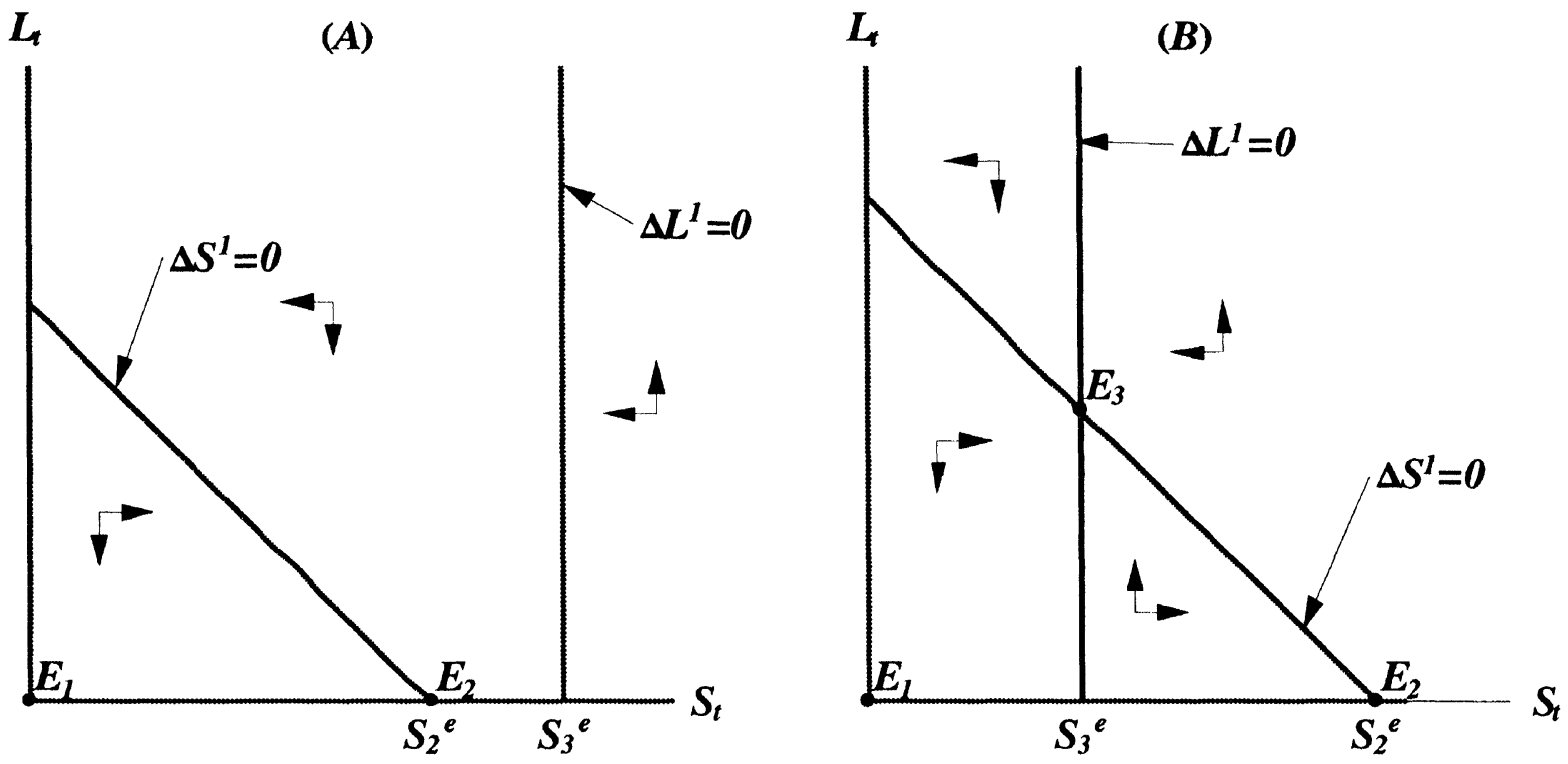

FIGURE 1 Determination of stationary state: (A) $K \alpha \beta \phi \leq n$ and (B) $K \alpha \beta \phi>n$.

This indicates that there are two $\Delta S=0$ loci on which $S_{t}$ is constant; one is $L_{t}=(r / \alpha \beta)-(r / \alpha \beta K) S_{t}$ which is denoted as $\Delta S^{1}=0$ and the other is $S_{t}=0$ which is denoted as $\Delta S^{2}=0$. On the nonnegative $\left(S_{t}, L_{t}\right)$ quadrant, the $\Delta S^{1}=0$ locus has a negative slope and crosses the horizontal axes at $S=K$ while the $\Delta S^{2}=0$ locus is identical with the vertical axis. By the second equation of Eq. (3), $\Delta L$ is

$$
\Delta L=\left(-n+\alpha \beta \phi S_{t}\right) \Delta t L_{t}
$$

This also implies that there are two $\Delta L=0$ loci on which $L_{t}$ is constant; one is $S_{t}=n / \alpha \beta \phi$ which is denoted as $\Delta L^{1}=0$ and the other is $L_{t}=0$ which is denoted as $\Delta \mathrm{L}^{2}=0$. The former locus is a vertical line crossing the horizontal axis at $S=n / \alpha \beta \phi$ while the latter is identical with the horizontal axis. As illustrated in Fig. 1, the nonnegative quadrant of $S_{t}$ and $L_{t}$ is partitioned into three regions by the $\Delta S^{1}=0$ and $\Delta L^{1}=0$ loci if $K \alpha \beta \phi \leq n$ and four regions if $K \alpha \beta \phi<n$. There it can be seen that the $\Delta S^{i}=0$ locus crosses the $\Delta L^{i}=0$ locus $(i=1,2)$ at each of the following three points;

$$
\left\{\begin{array}{l}
E_{1}=\left(S_{1}^{e}, L_{1}^{e}\right) \text { where } S_{1}^{e}=L_{1}^{e}=0 \\
E_{2}=\left(S_{2}^{e}, L_{2}^{e}\right) \text { where } S_{2}^{e}=K \text { and } L_{2}^{e}=0 \\
E_{3}=\left(S_{3}^{e}, L_{3}^{e}\right) \text { where } S_{3}^{e}=\frac{n}{\alpha \beta \phi} \text { and } L_{3}^{e}=\frac{r}{\alpha \beta}\left\{1-\frac{n}{K \alpha \beta \phi}\right\}
\end{array}\right.
$$

where $E_{1}$ is a solution of $\Delta S^{2}=\Delta L^{2}=0, E_{2}$ is a solution of $\Delta S^{1}=\Delta L^{2}=0$, and $E_{3}$ is a solution of $\Delta S^{1}=\Delta L^{1}=$ 0 . Since the loci of $\Delta S^{2}=0$ and $\Delta L^{1}=0$ are vertical lines, those do not intersect each other.

\section{DYNAMICS}

Arrows in Fig. 1 indicate directions in which variables $S_{t}$ and $L_{t}$ move. Those seem to suggest monotonic convergence to $E_{2}$ in Fig. $1 \mathrm{~A}$ and cyclic fluctuations around $E_{3}$ in Fig. 1B. To formally analyze dynamic behavior in a neighborhood of each of these three stationary points, we make Taylor expansion of the nonlinear dynamic system Eq. (3) and then check eigenvalues of the resultant Jacobi matrix. The Jacobi matrix evaluated at each of three stationary points is

$$
J_{i}=\left(\begin{array}{cc}
1+r \Delta t-2 \frac{r \Delta t}{K} S_{i}^{e}-\alpha \beta \Delta t L_{i}^{e} & -\alpha \beta \Delta t S_{i}^{e} \\
-\alpha \beta \phi \Delta t L_{i}^{e} & 1-n \Delta t+\alpha \beta \phi \Delta t S_{i}^{e}
\end{array}\right)
$$

where $i=1,2,3$. It is well known that the stationary state is asymptotically stable if and only if, for eigenvalues $\lambda_{1}$ and $\lambda_{2}$ of the Jacobi matrix, the following conditions hold,

$$
\left|\lambda_{1}\right|<1 \text { and }\left|\lambda_{2}\right|<1 \text {. }
$$

We will detect under what circumstances those stability conditions are satisfied.

\section{Stability of $E_{1}$ And $E_{2}$}

We start with stationary state $E_{1}$. Inserting $S_{1}^{e}=L_{1}^{e}=0$ into the Jacobi matrix Eq. (5) yields

$$
J_{1}=\left(\begin{array}{cc}
1+r \Delta t & 0 \\
0 & 1-n \Delta t
\end{array}\right)
$$

in which the eigenvalues are the diagonal elements $\lambda_{1}=$ 
$1+r \Delta t>1$ and $0<\lambda_{2}=1-n \Delta t<1$. Thus stationary state $E_{1}$ is a saddle point which a trajectory leaves on the horizontal axis to $E_{2}$ and approaches on the vertical axis to $E_{1}$ in the $\left(S_{t}, L_{t}\right)$ plane. To summarize, we have,

THEOREM $3 \quad E_{1}$ is an unstable saddle point for which the horizontal axis is an unstable manifold and the vertical axis is a stable manifold.

Turn to stationary point $E_{2}$. Inserting $S_{2}^{e}=K$ and $L_{2}^{e}=$ 0 of Eq. (4) into Eq. (5) yields the Jacobi matrix evaluated at $E_{2}$,

$$
J_{2}=\left(\begin{array}{cc}
1-r \Delta t & -\alpha \beta \Delta t K \\
0 & 1-n \Delta t+\alpha \beta \phi \Delta t K
\end{array}\right) .
$$

Its eigenvalues are also the diagonal elements $\lambda_{1}=$ $1-r \Delta t<1$ and $\lambda_{2}=1+(K \alpha \beta \phi-n) \Delta t>0$ in which the direction of inequality is due to Assumption. Since the positive growth rate of natural resource per the time-step has an upper bound (i.e. $0 \leq r \Delta t \leq 3$ ) by Theorem 2 , it can verified that

$$
\begin{gathered}
-1 \leq \lambda_{1} \leq 1 \text { if } 0<r \Delta t \leq 2 \text { and } \\
\lambda_{1}<-1 \text { if } 2<r \Delta t \leq 3 .
\end{gathered}
$$

Further, the following is straightforward,

$$
\lambda_{2}<1 \text { if } K \alpha \beta \phi \leq n \text { and } \lambda_{2}>1 \text { if } K \alpha \beta \phi>n .
$$

$E_{2}$ in Fig. $1 \mathrm{~B}$, in which $K \alpha \beta \phi>n$ holds, is definitely unstable as the dominant root is greater than unity whereas $E_{2}$ in Fig. 1A, in which $K \alpha \beta \phi \leq n$ holds, can be stable or unstable according to the natural growth rate over the time-step (i.e. $r \Delta t$ ) is less or greater than 2. Thus dynamic behavior in a vicinity of $E_{2}$ is summarized as follows.

THEOREM 4 (1) Provided that $K \alpha \beta \phi \leq n, E_{2}$ is a stable point if $0<r \Delta t \leq 2$ and a saddle point if $2<r \Delta t \leq 3$; (2) Provided that $K \alpha \beta \phi>n, E_{2}$ is a saddle point if $0<$ $r \Delta t \leq 2$ and an unstable point if $2<r \Delta t \leq 3$.

Theorem 4(1) indicates that

$$
K \alpha \beta \phi \leq n
$$

is the condition for extinction of humans (i.e. $L_{2}^{e}=0$ at $\left.E_{2}\right)$. We give an intuitive explanation for this condition. Since the endogenous fertility rate, $\phi(H / L)=S \alpha \beta \phi$, positively depends on the actual size of the natural resource, and $K$ is the carrying capacity of the natural stocks, $K \alpha \boldsymbol{\beta} \phi$ is the maximum fertility rate when the size of the natural resource reaches at its maximum (i.e. $S=K$ ). Since $n$ is the exogenous mortality rate of population, the inequality of Eq. (6) indicates that the maximum endogenous fertility rate is less than or equal to the exogenous mortality rate. Therefore, under this condition, the humans will extinct sooner or later regardless of their economic activities. This is what Theorem 4(1) implies. The stationary state $E_{3}$ is identical with $E_{2}$ if the equality of Eq. (6) holds whereas it becomes economically meaningless if the inequality of Eq. (6) strictly holds (i.e. $K \alpha \beta \phi<n \Rightarrow S_{3}^{e}>K$ and $L_{3}^{e}<0$ )."

\section{Stability of $E_{3}$}

Since we are interested in the survival of humans, we confine the following analysis to a case in which the maximum endogenous fertility rate is greater than the exogenous mortality rate,

$$
K \alpha \beta \phi>n
$$

which we call the survival condition. The consistent requirement, the natural resource is less than the carrying capacity (i.e. $S_{3}^{e}<K$ ) and the population size is positive (i.e. $L_{3}^{e}>0$ ), holds under the survival condition. The population size can increase or decrease depending on the relative magnitude between the actual fertility rate, $\alpha \beta \phi S_{t}$, and the exogenous mortality rate, $n$. This is a source of dynamics in our model.

Inserting $\left(S_{3}^{e}, L_{3}^{e}\right)$ into Eq. (5) yields the Jacobi matrix evaluated at the stationary point $E_{3}$,

$$
J_{3}=\left(\begin{array}{cc}
1-\frac{n r \Delta t}{K \alpha \beta \phi} & -\frac{n \Delta t}{\phi} \\
r \Delta t\left(\phi-\frac{n}{K \alpha \beta}\right) & 1
\end{array}\right) .
$$

Its characteristic polynomial is $f(\lambda)=\lambda^{2}-\operatorname{tr} J_{3} \lambda+$ $\operatorname{det} J_{3}$ and its eigenvalues are roots of $f(\lambda)=0$,

$$
\lambda_{1,2}=\frac{\operatorname{tr} J_{3} \pm \sqrt{\left(\operatorname{tr} J_{3}\right)^{2}-4 \operatorname{det} J_{3}}}{2}
$$

where

$$
\begin{aligned}
& \operatorname{tr} J_{3}=\lambda_{1}+\lambda_{2}=2-\frac{n r \Delta t}{K \alpha \beta \phi}, \\
& \operatorname{det} J_{3}=\lambda_{1} \lambda_{2}=\frac{K \alpha \beta \phi\left(1+n r \Delta t^{2}\right)-n r \Delta t(1+n \Delta t)}{K \alpha \beta \phi} .
\end{aligned}
$$

The roots are real if the discriminant $D=\left(\operatorname{tr} J_{3}\right)^{2}-$ $4 \operatorname{det} J_{3}$ is nonnegative and complex if $D$ is negative. Thus points on the $D=0$ locus construct a line of demarcation between real roots and complex roots. Given $r$, solving

\footnotetext{
"After the humans become extinct (i.e. $L_{t}=0$ for all $t$ ), the natural resource will develop according to the following logistic map, $x_{t+1}=a\left(1-x_{t}\right) x_{t}$ where $x_{t}=(r \Delta t) /(1+r \Delta t) K$ and $a=1+r \Delta t$. If $a \leq 3$ or $r \Delta t \leq 2$, then natural resource gradually grows and reaches at its carrying capacity. Although it has been well known that the logistic map can generate chaotic dynamic for $a>3$ or $r \Delta t>2$, the economic constraint $S_{t} \leq K$ prevent the logistic map generating such complex dynamic. Numerical simulations indicate that trajectories of $S_{t}$ subject to $S_{t} \leq K$ reaches $K$ within finite time periods even if $r \Delta t>2$ holds.
} 
$D=0$ for $K \alpha \beta \phi$ yields the demarcation line,

$$
f_{\mathrm{dem}}(n)=\frac{n+\sqrt{n(n+r)}}{2} .
$$

The stability condition, both eigenvalues are less than unity in absolute values, is hold if and only if the following inequalities are satisfied, ${ }^{\#}$

$$
-1 \pm \operatorname{tr} J_{3}<\operatorname{det} J_{3}<1
$$

where the first inequality ensures that the real roots lie in the interval $(-1,1)$ and the second ensures the modulus of the complex roots is less than unity. To find the stable region, we construct the lines $f(1)=0, f(-1)=0$ and $\operatorname{det} J_{3}-1=0 \quad$ where $f(1)=1-\operatorname{tr} J_{3}+\operatorname{det} J_{3} \quad$ and $f(-1)=1+\operatorname{tr} J_{3}+\operatorname{det} J_{3}$. Substituting the most right expressions of Eq. (8) into each of those lines gives

$$
\left\{\begin{array}{l}
f(1)=\frac{n r \Delta t^{2}}{K \alpha \beta \phi}(K \alpha \beta \phi-n), \\
f(-1)=\frac{4+(n \Delta t)(r \Delta t)}{K \alpha \beta \phi}\left\{K \alpha \beta \phi-n \frac{2 r \Delta t+(n \Delta t)(r \Delta t)}{4+(n \Delta t)(r \Delta t)}\right\} \\
\operatorname{det} J_{3}-1=\frac{n r \Delta t^{2}}{K \alpha \beta \phi}\left\{K \alpha \beta \phi-\left(n+\frac{1}{\Delta t}\right)\right\} .
\end{array}\right.
$$

Therefore, given $r$ and $\Delta t$, the stable region of the stationary state $E_{3}$ in the $(n, K \alpha \beta \phi)$ plane is surrounded by the following boundary lines,

$f(1)=0 \Leftrightarrow K \alpha \beta \phi=f_{\text {div }}(n) \equiv n$,

$f(-1)=0 \Leftrightarrow K \alpha \beta \phi=f_{\text {flip }}(n) \equiv n \frac{2 r \Delta t+(n \Delta t)(r \Delta t)}{4+(n \Delta t)(r \Delta t)}$,

$\operatorname{det} J_{3}=1 \Leftrightarrow K \alpha \beta \phi=f_{\text {flut }}(n) \equiv n+\frac{1}{\Delta t}$,

which we call the divergence boundary, the flip boundary and the flutter boundary, respectively, following Sonis (1996). Since it can be shown that

$$
f_{\text {flip }}(n) \frac{<}{>} f_{\text {div }}(n) \text { according to } r \Delta t \frac{<}{>},
$$

two different stable regions are, under the survival condition defined by

$$
\begin{aligned}
\mathbb{M}_{1} & =\left\{(n, K \alpha \beta \phi) \mid 0 \leq n, f_{\text {div }}(n)<K \alpha \beta \phi\right. \\
& \left.<f_{\text {flut }}(n)\right\} \text { if } r \Delta t \leq 2, \\
\mathbb{M}_{2} & =\left\{(n, K \alpha \beta \phi) \mid 0 \leq n, f_{\text {flip }}(n)<K \alpha \beta \phi\right. \\
& \left.<f_{\text {flut }}(n)\right\} \text { if } r \Delta t>2 .
\end{aligned}
$$

To summarize the dynamic behavior in a vicinity of the stationary state $E_{3}$, we have:
THEOREM 5 (1) Provided that $r \Delta t \leq 2, E_{3}$ is stable for $(n, K \alpha \beta \phi) \in \mathbb{M}_{1} ;(2)$ provided that $r \Delta t>2, E_{3}$ is stable for $(n, K \alpha \beta \phi) \in \mathbb{M}_{2}$.

$\mathbb{N}$ in Theorem 2 concerns the feasibility of solutions as it is the set of parameters $(n, K \alpha \beta \phi)$ for which $F(\mathbb{R}) \subset \mathbb{R} . \mathbb{M}_{i}$ in Theorem 5 concerns the stability of solutions as it is the set of parameters $(n, K \alpha \beta \phi)$ for which $F(\mathbb{R})$ generates stable dynamics. Thus an intersection of $\mathbb{M}_{i}$ and $\mathbb{N}$ is the set of parameters for which solutions of $F(\mathbb{R})$ are feasible and stable. Returning to the definition of the set $\mathbb{N}$, we can see that the upper boundary of $\mathbb{N}$ in the $(n, K \alpha \beta \phi)$ plane is

$$
f_{\text {up }}(n) \equiv \frac{r}{1+r \Delta t}(1+\sqrt{n \Delta t})^{2},
$$

which is defined on the interval $[0,1 / \Delta t\rfloor$. To check whether this upper boundary and the flutter boundary intersect, we solve $f_{\text {up }}(n)=f_{\text {flut }}(n)$ for $n$ to have

$$
n_{1,2}=\frac{2(r \Delta t)^{2}-1 \pm 2 r \Delta t \sqrt{(r \Delta t)^{2}-1}}{\Delta t} .
$$

This implies that the upper boundary is tangent to the flutter boundary at $(1 / \Delta t, 2 / \Delta t)$ if $r \Delta t=1$. Thus the upper boundary of $\mathbb{N}$ and the flutter boundary of $\mathbb{M}_{i}$ intersect none if $r \Delta<1$ and once in the interval $(0,1 / \Delta t)$ if $r \Delta t>1$.** By Theorem 2, we have $0<r \Delta t \leq 3$. By Theorem 5 , the stable set is either $\mathbb{M}_{1}$ or $\mathbb{M}_{2}$ according to $r \Delta t<2$ or $r \Delta t>2$. Summing up the foregoing discussions indicates that for detecting the dynamics behavior of $E_{3}$, three distinct cases can be identified which depend on the growth rate of natural resource over the time-step: (1) $0 \leq r \Delta t \leq 1$, (2) $1<r \Delta t \leq 2$ and (3) $2<r \Delta t \leq 3$. Dynamics in each of three cases is considered, allowing for the constraints imposed on parameters,

$$
0<n \Delta t \leq 1 \text { and } K \alpha \beta \phi>n .
$$

Case $10 \leq r \Delta t \leq 1$. In this case, $f_{\text {up }}(n) \leq f_{\text {flut }}(n)$ for all $n \in[0,1 / \Delta t]$. Thus the upper bound of the $\mathbb{N} \cap \mathbb{M}_{1}$ is the $K \alpha \beta \phi=f_{\text {up }}(n)$ locus. The dynamics of $E_{3}$ is summarized as follows.

THEOREM 6 Provided that $0 \leq r \Delta t \leq 1, E_{3}$ is stable for $(n, K \alpha \beta \phi) \in \mathbb{N} \cap \mathbb{M}_{1}=\{(n, K \alpha \beta \phi) \mid 0 \leq n \leq 1 / \Delta t, n<$ $\left.K \alpha \beta \phi \leq r /(1+r \Delta t)(1+\sqrt{n \Delta t})^{2}\right\}$.

In Fig. 2 below, the stable and feasible region $\mathbb{N} \cap \mathbb{M}_{1}$ is depicted as an shaded area surrounded by the upper boundary, the divergence boundary, the vertical axis and the vertical line, $n=1 / \Delta t$. It is divided into two regions by the demarcation line on which we have a real root of multiplicity two. For any point in a region below the demarcation line and above the divergence boundary (i.e. the dark-shaded region), the eigenvalues are real so that

\footnotetext{
"See Gandolfo (1997) for a heuristic proof.

**In particular, two boundaries cross twice for $n=n_{1}$ and $n=n_{2}$ if $r \Delta t>1$. We can assume $n_{1}<n_{2}$ without a loss of generality which implies $n_{1}<1 / \Delta t<n_{2}$. However, $f_{\text {up }}(n)$ is defined only on the interval $[0,1 / \Delta t]$ so that we do not need to consider the second intersection for $n=n_{2}$.
} 


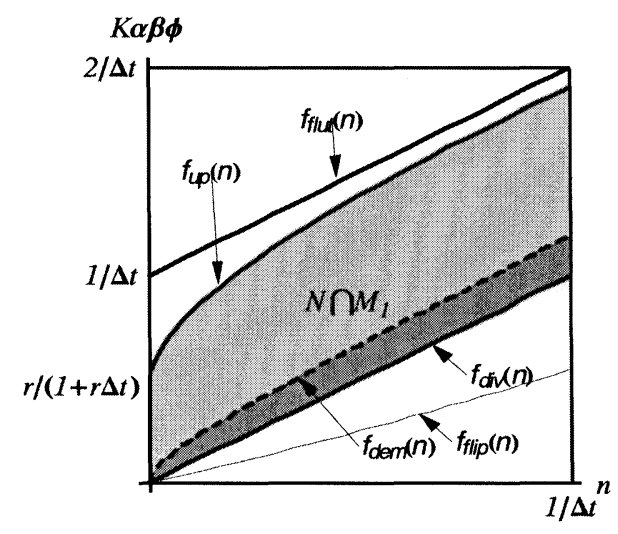

FIGURE 2 Stable and feasible region; $0 \leq r \Delta t \leq 1$.

the dynamic system $F(\mathbb{R})$ shows a monotonic convergence toward the stationary state $E_{3}$. On the other hand, for any point in a region above the demarcation line and below the upper boundary (i.e. the light-shaded region), the eigenvalues are complex so that $F(\mathbb{R})$ shows a damped oscillations around $E_{3}$.

Using those results together with Theorem 6, the following is implied.

COROllary 7 For $(n, K \alpha \beta \phi) \in \mathbb{N} \cap \mathbb{M}_{1}, E_{3}$ is a stable node with monotonic convergence if $K \alpha \beta \phi \leq(n+$ $\sqrt{n(n+r))} / 2$ and a stable focus with damped oscillations if $(n+\sqrt{n(n+r)}) / 2<K \alpha \beta \phi$.

Theorems 3-5 and Corollary 7 imply that the discretetime system can generate the similar dynamics as the continuous-time system if the time step, $\Delta t$, is small enough in a sense that $\Delta t$ is smaller than an inverse of $r$. Dynamics of the stationary states in the continuous-time model is summarized in Theorem 1 above. Theorem 3, which shows that the stationary state $E_{1}$ is a saddle point, is the same as Theorem 1(i). The first part of Theorem 4(2), which shows that the stationary state $E_{2}$ is an unstable saddle point, is the same as Theorem 1(ii). Lastly, Corollary 7 just mentioned above is discrete-time counterparts of Theorem 1(iii) and (iv). ${ }^{\dagger \dagger}$ Thus BT's results follow in the discrete-time model.

It is possible to show that the discrete-time model can generate qualitatively different dynamics. To this end, we simulate the model, using parameter values used by BT. The parameter values used in BT can be divided into two; one is a set of time-independent parameters that can be used either of continuous- or discrete-time model and the other is a set of time-dependent parameters that are sensitive to a choice of continuous- or discrete-time model. The carrying capacity of the island, $K$, the labor harvesting productivity, $\alpha$, measure of preference for the output of the harvest good, $\beta$, and a constant factor of the fertility function, $\phi$, are thought to be in the former set so that we coax parameter values from BT and set $K=$ $12,000, \alpha=0.00001, \beta=0.4$ and $\phi=4$ in the following simulations. The exogenous motility rate, $n$, and the intrinsic growth rate of natural resource, $r$, are in the latter set and, as will be seen shortly, play critical roles for the emergence of complex dynamics. We take those to be $n=0.05$ and $r=0.04$ in the first simulation. Further, we set $\Delta t=10$ and take the initial values of $S_{t}$ and $L_{t}$ as $S_{0}=K$ and $L_{0}=100$. As a result of those specifications, a pair of $K \alpha \beta \phi=0.192$ and $n=0.05$ are not in $\mathbb{N} \cap \mathbb{M}_{1}{ }^{19}$ Although the stationary state is unstable, the simulation illustrated in Fig. 3A shows an aperiodic oscillation that the continuous-time model cannot generate. As plotted in Fig. 3B, evolutions of the natural resource (the dotted line) and the population (the real line) would resemble to the "history" of Easter Island.

Following BT, we assume that the history of Easter Island starts shortly after 400 A.D. and ended in the 1800 s by the outside intervention. A growth-collapse pattern during those 1400 years are fairly described by the simulation if we presume that a time-step equals 100 actual years (i.e. $\Delta t=100$ years where $\Delta t=10$ time-units indicate that one-time unit is one decade). In particular, in the simulation, after 4 periods of the beginning of the history (i.e. about 800 A.D. in the actual calendar), the natural resource degradation starts and population begins to increase rapidly. At 9th period (i.e. at 1100 A.D.), population peaks at $L=30,000$ unity that means that 15,000 people live on the island while the natural resource is degraded severely. At 12th period (i.e. 1600 A.D.) the natural resource was almost gone to nothing and population continues to decline to 2500 from its peak. It is said that the carving stone statues starts around 1100 A.D after the size of population becomes larger than the essential size and stops around 1600 A.D due to the extinction of palm tree forest on the island. Such archeological evidence can go with the simulation results. At the 14th period (i.e. 1800A.D.) the history of endogenous evolution ended. This simulation mimics a feast (i.e. a gradual-rapid population growth and intensive use of natural resource) and famine (i.e. rapid population collapse and environmental degradation) pattern, which is often observed in small island economy. In the simulation $r \Delta t=0.4$ and $n \Delta t=0.5$ which means that the natural resource increases by $40 \%$ per century (i.e. $4 \%$ per decade) without human harvesting and the base motility rate per century is $50 \%$ ( $5 \%$ per decade), both of which are supposed to be plausible estimates.

Case $21<r \Delta t \leq 2$. Case 2 deals with the effect caused by an increase in the intrinsic growth rate per unit time interval on the dynamic behavior. The first inequality condition $1<\Delta t$ makes the discriminant of Eq. (12) positive, that is, two roots, $n_{1}$ and $n_{2}$, are real and distinct.

\footnotetext{
${ }^{\dagger}$ An alternative expression of $(n+\sqrt{n(n+r)}) / 2<K \alpha \beta \phi$ is $n r / K \alpha \beta \phi+4(n-K \alpha \beta \phi)<0$, which is Eq. (14) of BT.

Nwo unit of labor force is assumed to be one person. Thus $L_{0}=100$ means that the founding population is 50 .

${ }^{11}$ Since Theorem 2 provides a sufficient condition for feasibility, it is possible that a trajectory starting at a point outside of $\mathbb{N}$ oscillates around an unstable stationary point if the non-linearity's of the model are strong enough.
} 

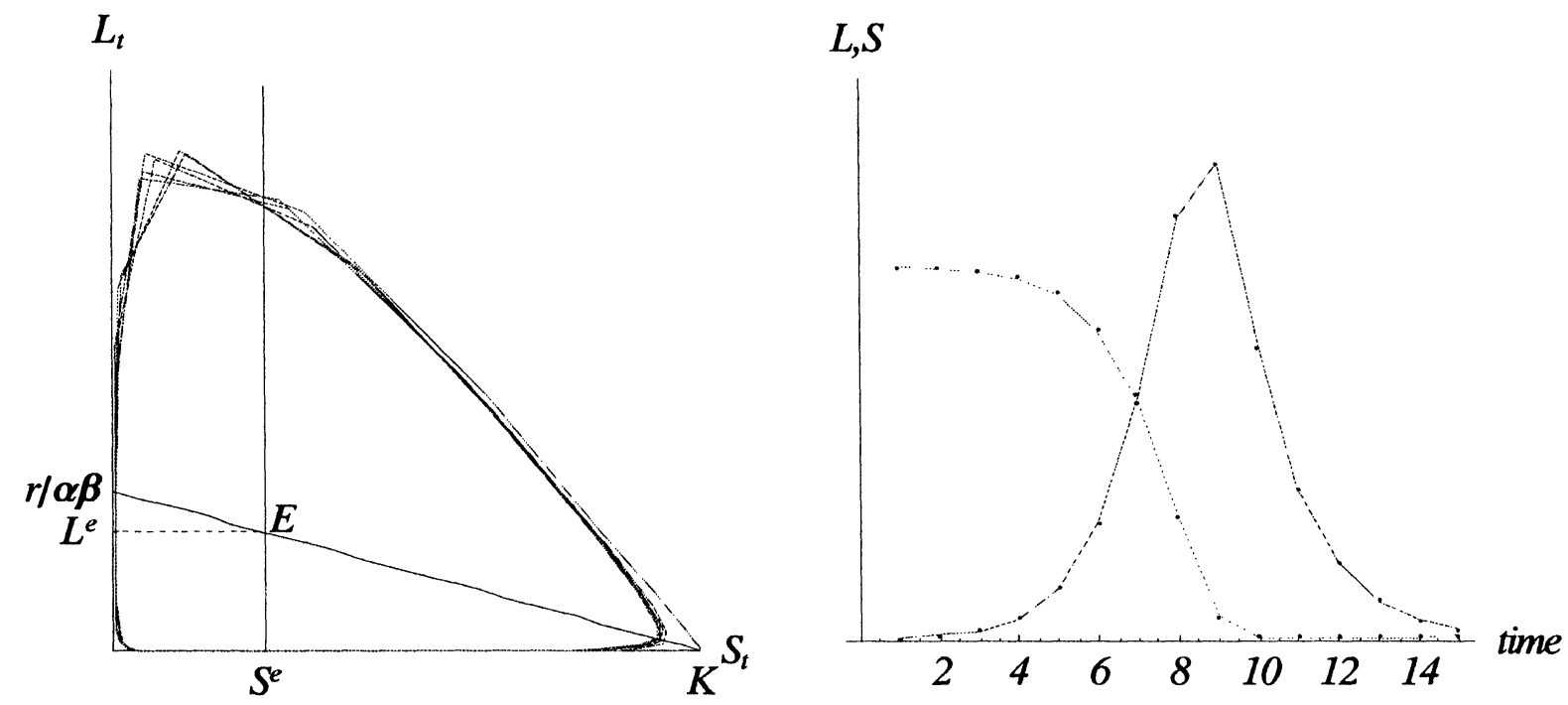

FIGURE 3 Numerical simulation of Easter Island evolution.

The upper boundary and the flutter boundary intersect for $n=n_{1}<1 / \Delta t$. Consequently the upper bound of the feasible and stable region is $\min \left[f_{\text {up }}(n), f_{\text {flut }}(n)\right]$ in which $f_{\text {up }}(n)<f_{\text {flut }}(n)$ holds for $0<n<n_{1}$ and the direction of inequality is reversed for $n_{1}<n \leq 1 / \Delta t$. Further, since the second inequality condition $r \Delta t \leq 2$ leads to $f_{\text {flip }}(n) \leq$ $f_{\text {div }}(n)$ the divergence boundary is the lower bound of $\mathbb{M}_{1}$. Thus, in Case 2, the feasible and stable region is an intersection of $\mathbb{N}$ and $\mathbb{M}_{1}$.

$$
\begin{aligned}
& \mathbb{N} \cap \mathbb{M}_{1}=\left\{(n, K \alpha \beta \phi) \mid 0 \leq n \leq \frac{1}{\Delta t},\right. \\
&\left.f_{\text {div }}(n) \leq K \alpha \beta \phi \leq \min \left[f_{\text {up }}(n), f_{\text {flut }}(n)\right]\right\} .
\end{aligned}
$$

Since $f_{\text {flut }}(n)<f_{\text {up }}(n)$ for $n>n_{1}$, we can define a feasible and unstable region denoted as $\mathbb{U}_{1}$,

$$
\begin{aligned}
\mathbb{U}_{1}=\left\{(n, K \alpha \beta \phi) \mid n_{1} \leq n \leq \frac{1}{\Delta t},\right. \\
\left.\left.f_{\text {flut }}(n) \leq K \alpha \beta \phi \leq f_{\text {up }}(n)\right]\right\} .
\end{aligned}
$$

The feasible and stable region (i.e. a dark and light shaded region) and the feasible and unstable region (i.e. lighter shaded region) are depicted in Fig. 4.

Thus the results are summarized as

THEOREM 8 Provided that $1<r \Delta t \leq 2, E_{3}$ is stable for $(n, K \alpha \beta \phi) \in \mathbb{N} \cap \mathbb{M}_{1}$ and unstable for $(n, K \alpha \beta \phi) \in \mathbb{U}_{1}$
For $(n, K \alpha \beta \phi) \in \mathbb{N} \cap \mathbb{M}_{1}$, the dynamic generated in Case 2 is qualitatively the same as the one in Case 1. A trajectory exhibits either a monotonic or oscillatory movement converging towards the stationary state $E_{3}$ according to the roots of the characteristic equation are real or complex. For $(n, K \alpha \beta \phi) \in \mathbb{U}_{1}$, a trajectory generated by Eq. (3) does not converge to the stationary state since the stability condition is violated. In spite of this instability, the trajectory does not explode globally but keeps oscillating in a limited region due to inherent nonlinearity's in the model. To see what happens in the region $\mathbb{U}_{1}$, we have to examine the points on the flutter boundary on which eigenvalues are complex and $\left|\lambda_{1}\right|=$ $\left|\lambda_{2}\right|=1$ where $\operatorname{det} J_{3}=\lambda_{1} \lambda_{2}$ is equal to the modulus of roots. The stationary point is nonhyperbolic so that the non-linear model and its linear approximation are not topologically equivalent in a vicinity of $E_{3}$. Crossing of the flutter boundary means that the second- and higherorder derivatives of the behavioral functions becomes essential and creates a Hopf bifurcation from the stationary state. To detect the dynamic behavior, we simulate the model choosing the mortality rate as a bifurcation parameter and fixing other parameters at some appropriate values. ${ }^{\S \S}$ Figure 5 below presents the bifurcations appearing when $n$ is moving along a straight line $a \bar{b}$ from $n_{b}$ to $n_{a}$ in the region $\mathbb{U}_{1}$ in Fig. $4 .{ }^{\text {IIII }}$ For each value of $n$, the dynamic system starts from the same initial values, $S_{0}=K$ and $L_{0}=100$ and is simulated for 20 iterations to see the long-run dynamic behavior. It will be safe to assume one period equal one hundred years as in the first simulation. It can be seen that trajectories goes more sharply ups and downs, as $n$ gets smaller than $n_{b}$. This indicates that a change from a heyday to a hell-day in

\footnotetext{
${ }^{\S \S}$ As in the first simulation, we set $\alpha=0.00001, \beta=0.4, \phi=4, K=12,000$ which leads to $K \alpha \beta \phi=0.192$ and take $r$ and $\Delta t$ to be 0.15 and 10 which leads to $1<r \Delta t \leq 2$.

\|\|$\|_{\text {Line }} a \bar{b}$ is divided into ten segment by points defined as $n(i)=n_{b}-(i / T)\left(n_{b}-n_{a}\right), i=0,1, \ldots T$ where $T$ is the number of bifurcation steps. $T=10$ in the simulation. See Sonis (1996) for more precise explanations for this bifurcation cascade.
} 


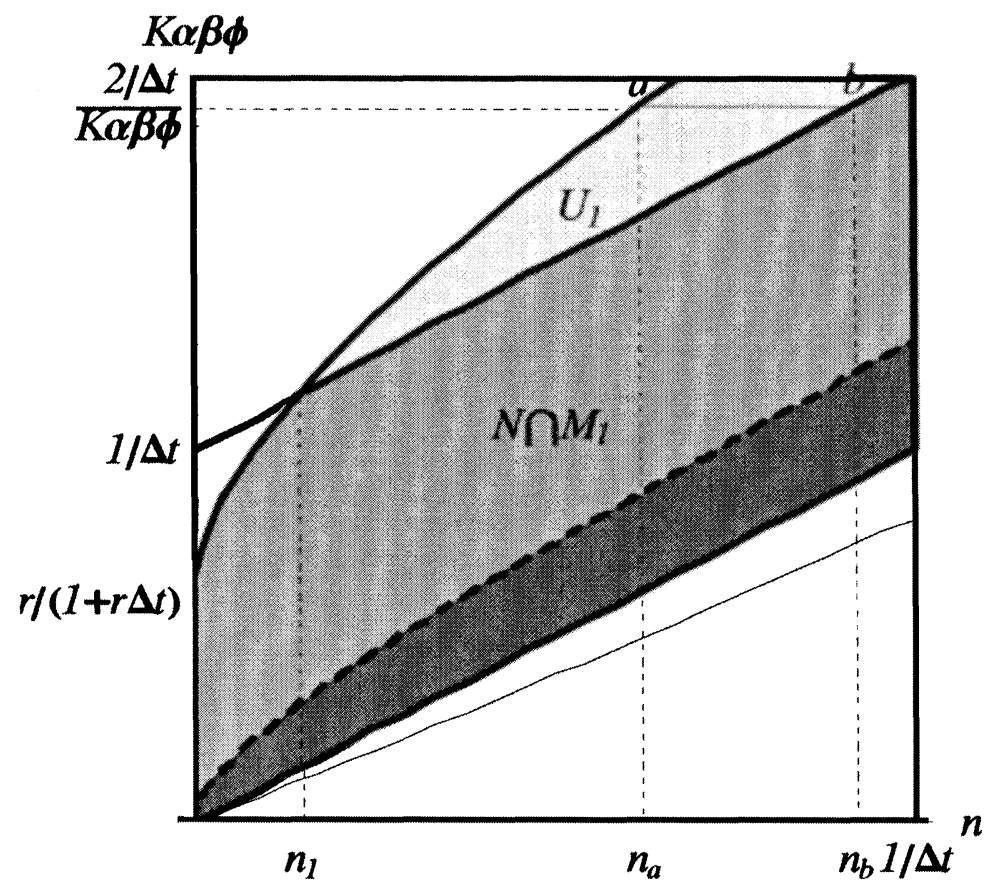

FIGURE 4 Stable and feasible region: $1<\Delta t \leq 2$.

a feast and famine cycle gets more rapid and drastic as the exogenous mortality rate becomes smaller.

Case $32<r \Delta t \leq 3$. Suppose $r \Delta t$ becomes larger and lies in an interval $(2,3]$. As will be seen shortly, the higher intrinsic growth rate of natural resource increases a possibility causing complex long-run dynamics. Similar to Case 2, the upper boundary and the flutter boundary intersect at $n=n_{1}$ and thus $f_{\text {up }}(n)<f_{\text {flut }}(n)$ holds for $0<$ $n<n_{1}$ and $f_{\text {up }}(n)>f_{\text {flut }}(n)$ holds for $n_{1}<n \leq 1 / \Delta t$. In addition to this, the flip boundary gets steeper than the divergence boundary for $r \Delta t>2$. Thus the feasible and stable region is defined by an intersection of $\mathbb{N}$ and $\mathbb{M}_{2}$,

$$
\begin{aligned}
\mathbb{N} \cap \mathbb{M}_{2}= & \left\{(n, K \alpha \beta \phi) \mid 0 \leq n \leq \frac{1}{\Delta t},\right. \\
& \left.f_{\text {flip }}(n) \leq K \alpha \beta \phi \leq \min \left[f_{\text {up }}(n), f_{\text {flut }}(n)\right]\right\} .
\end{aligned}
$$

The unstable region $\mathbb{U}_{1}$ is similarly defined as a region between the upper boundary and the flutter boundary for $n \geq n_{1}$. Since $E_{3}$ is economically feasible only for $K \alpha \beta \phi>f_{\text {div }}(n)$ (i.e. the survival condition, $K \alpha \beta \phi>n$ ),
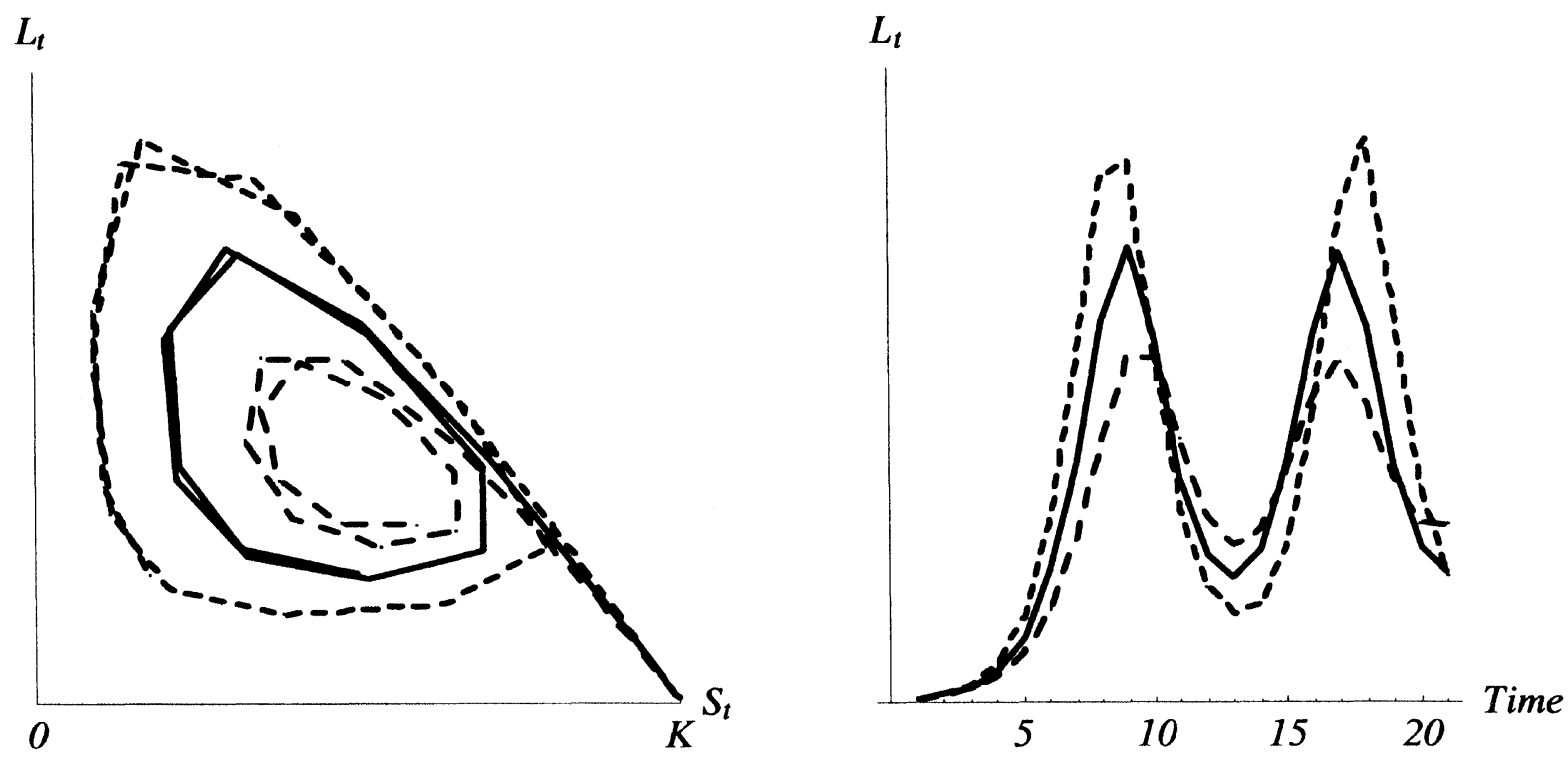

FIGURE 5 Various oscillations. 


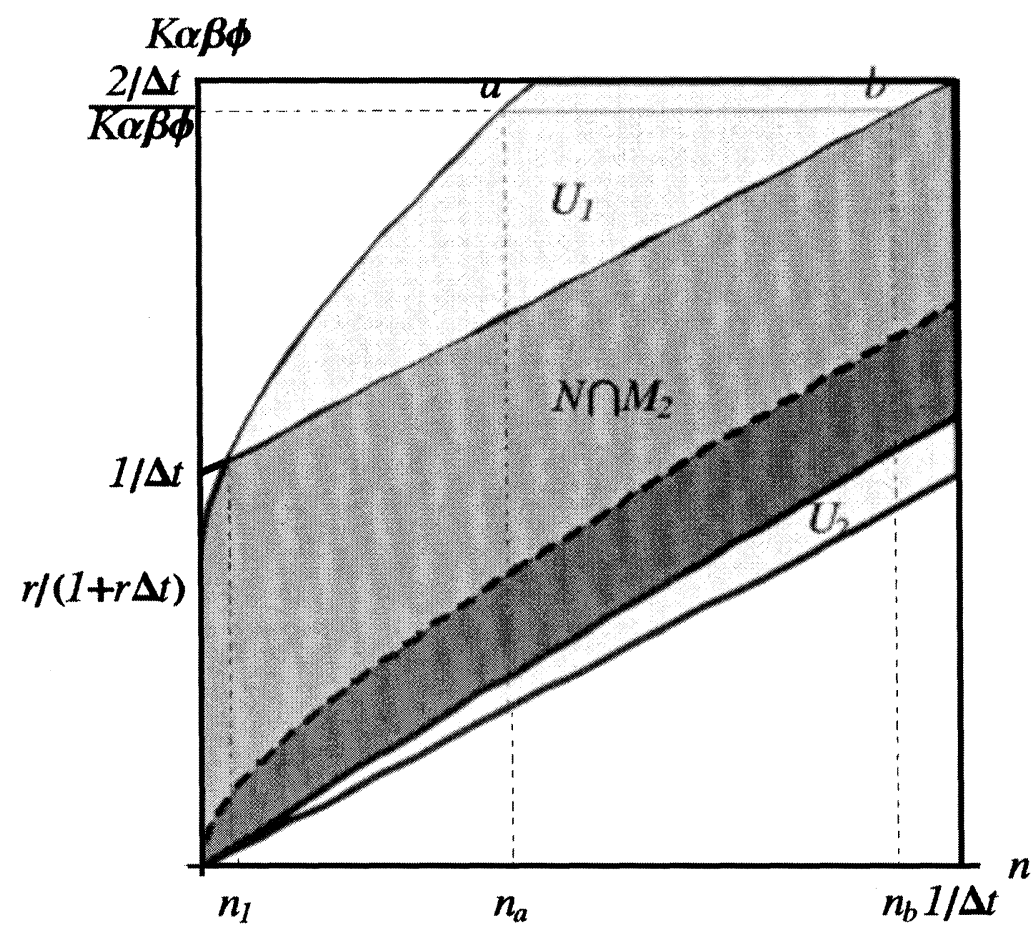

FIGURE 6 Stable and unstable regions; $2<\Delta t \leq 3$.

another feasible and unstable region, denoted as $\mathbb{U}_{2}$, can be defined as:

$$
\begin{aligned}
\mathbb{U}_{2}= & \left\{(n, K \alpha \beta \phi) \mid 0 \leq n \leq \frac{1}{\Delta t},\right. \\
& \left.\left.f_{\text {div }}(n)<K \alpha \beta \phi \leq f_{\text {flip }}(n)\right]\right\} .
\end{aligned}
$$

Thus the results are summarized as

THEOREM 9 Provided that $2 \leq r \Delta t \leq 3, E_{3}$ is stable for $(n, K \alpha \beta \phi) \in \mathbb{N} \cap \mathbb{M}_{2}$ and unstable for $(n, K \alpha \beta \phi) \in$ $\mathbb{U}_{1} \cup \mathbb{U}_{2}$.

When the stationary state is stable, dynamic is similar to the one considered in Case 1 and Case 2, that is, monotonic convergence or damped oscillations. When the stationary state is unstable, the non-linear structure of the model results in far more complex dynamics for which there is no general theoretical results. Numerical simulation is useful to detect such non-linear structure. Crossing of the flutter boundary implies the start of Hopf bifurcation cascade to chaos. In particular, the third simulation is done with $r=2.5$ and the exogenous motility rate is chosen as a bifurcation parameter. As $n$ decreases from $n_{b}$ which is on the flutter boundary, the stationary state bifurcates to limit cycles, which represents an increase in the magnitude of population ups and downs. However, the cycles are relatively regular and (quasi-) symmetrical as illustrated in Fig. 7A. As $n$ decreases more, the cycles are distorted and show irregular oscillations. As $n$ gets to $n_{a}$, chaotic or very high-period cycles appears as seen in Fig. 7B in which chaotic attractor is represented.

Crossing of the flip boundary makes $E_{3}$ unstable and generates a period-doubling bifurcation, which is a typical route to chaos. A periodic cycle with period two emerges for each point on the flip boundary. ${ }^{\# \#}$ Although $S_{t}$ exhibits various dynamics from periodic cycles to chaotic movement, it becomes larger than the carrying capacity for some values of $n$. Thus such dynamic behavior is economically unrealistic. If we perform simulation allowing for the capacity constraint $S_{t} \leq K$ explicitly, then high order cycles including chaotic behavior disappear but a two-periodic cycle appears.

\section{CONCLUDING REMARKS}

In this study, we have demonstrated that a discrete version of BT's continuous-time model for small islands can generate various dynamics ranging from stable as well as periodic trajectories to complex dynamics involving chaos. In particular, on the one hand, Theorem 4 shows a natural result that the humans are extinct soon or later if the maximum fertility rate is less than mortality rate, $K \alpha \beta \phi \leq n$. On the other hand, Theorems 5-9 shows that sources of a diversity of dynamics are degrees of the intrinsic growth rate of natural resource over a unit of time $r \Delta t$ and the base mortality rate, in a unit of time $n \Delta t$ even if the maximum fertility rate is greater than the mortality

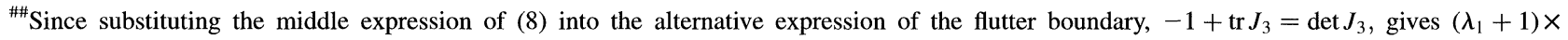
$\left(\lambda_{2}+1\right)=0$, one of the eigenvalues is -1 and the other is $-\operatorname{det} J_{3}$ on the flip boundary.
} 

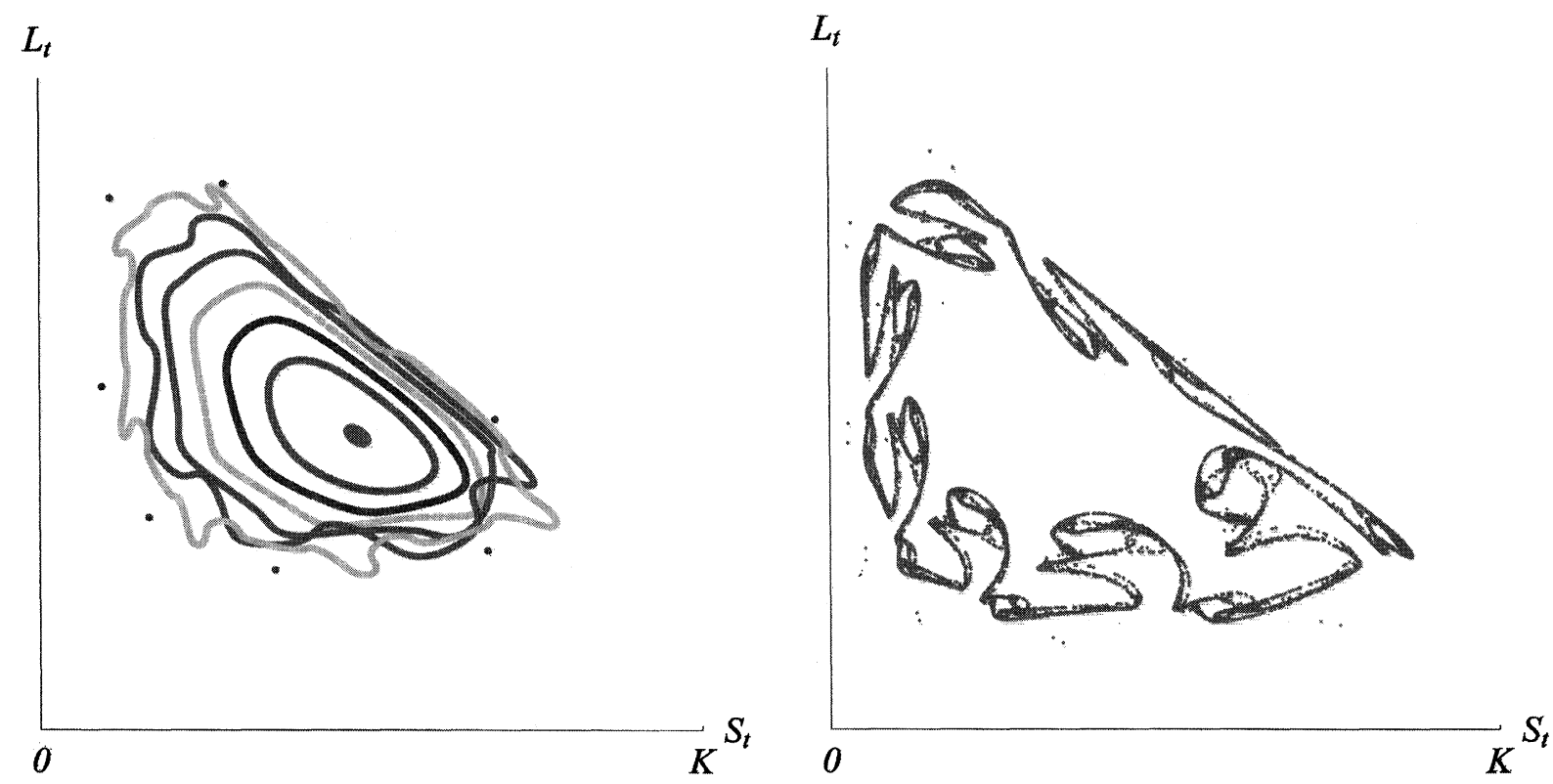

FIGURE 7 (A) invariant cycles, (B) strange attractor.

rate, $K \alpha \beta \phi>n$. To summarize our results, we can say that a discrete-time model can be a good candidate for explaining various pattern of evolutions observed in small islands.

\section{Acknowledgements}

I would appreciate Professors M. Okamaura, T. Asada and T. Onozaki for helpful comments and CRUGE of Chuo University for financial supports.

\section{References}

Brander, J.A. and Taylor, N.S. (1998) "The simple economics of Easter Island: A Ricardo-Malthus model of renewable resource use", American Economic Review 88, 119-138.

Daltion, T.R. and Coats, R.M. (2000) "Could institutional reform have saved Easter Island", Journal of Evolutionary Economics 10, 489-505.

Erickson, J.D. and Gowdy, J.M. (2000) "Resource use, institutions, and sustainability: a tale of two Pacific Island cultures", Land Economics 76, 345-354.

Gandolfo, G. (1997) Economic Dynamics (Springer, Berlin).

Intoh, M. (2000) "Prehistorical Oceania", in Oceania History ed., by M. Yamamoto, Yamakawa.

Pezzy, J.C.V., Anderies, J.M. (2000). "Some further Economics of Easter Island: Adding Subsistence and Resource Conservation", mimeo, Oct., 2000.

Sonis, M. (1996) "One more on Henon map: analysis of bifurcations", Chaos, Solitons and Fractals 7, 2215-2234.

\section{APPENDIX}

In this appendix, we show that $F(\mathbb{R}) \subset \mathbb{R}$ if $0 \leq r \Delta t \leq 3$ and $(n, K \alpha \beta \phi) \in \mathbb{N}$. A boundary line of $\mathbb{R}, L=g(S)$, is downward sloping and crosses the vertical axis at $L_{0}=$ $1+r \Delta t / \alpha \beta \Delta t$ and the horizontal axis at $S_{0}=(1+$ $r \Delta t) /(r \Delta t) K$. As a first step, we construct a locus of $S$ and $L$ such that $\{(S, L) \mid S \geq 0, L \geq 0$ and $\operatorname{det} J=0\}$ where $\operatorname{det} J$ is the determinant of the Jacobi matrix of the dynamic system Eq. (3),

$J=\left(\begin{array}{cc}1+r \Delta t-2 \frac{r \Delta t}{K} S_{t}-\alpha \beta \Delta t L_{t} & -\alpha \beta \Delta t S_{t} \\ -\alpha \beta \phi \Delta t L_{t} & (1-n \Delta t)+\alpha \beta \Delta t S_{t}\end{array}\right)$

Solving $\operatorname{det} J=0$ for $L$ yields a critical line, denoted as $g(S)$, that divides $\mathbb{R}$ into two regions $\mathbb{R}_{1}$ and $\mathbb{R}_{2} ; \operatorname{det} J>0$ in $\mathbb{R}_{1}$ and $\operatorname{det} J<0$ in $\mathbb{R}_{2}$,

$$
h(S)=\frac{\left[K(1+r \Delta t)-2 r \Delta t S_{t}\right]\left[(1-n \Delta t)+\alpha \beta \Delta t S_{t}\right]}{(1-n \Delta t) K \alpha \beta \Delta t}
$$

As illustrated in Fig. A1, the $L=h(S)$ curve crosses the horizontal axes at point $A=\left(S_{A}, L_{A}\right)$ where $S_{A}=S_{0} / 2$, $L_{A}=0$ and the vertical axes at point $B=\left(S_{B}, L_{B}\right)$ where $S_{B}=0$ and $L_{B}=L_{0}$. The next step is to compute an intersection(s) of those two curves, $L=f(S)$ and $L=g(S)$. It can be verified that, in the non-negative quadrant of ( $S$, $L)$, two curves intersect once at point $B$ if $K \alpha \beta \phi \leq r(1-$ $\alpha \beta \phi \leq r(1-n \Delta t) /(1+r \Delta t)$ and twice at point $B$ and point $C=\left(S_{c}, L_{c}\right)$ where $S_{c}=[(1+r \Delta t) K \alpha \beta \phi-r(1-$ $n \Delta t)] / 2 r \alpha \beta \phi \Delta t \quad$ and $\quad L_{c}=[(1+r \Delta t) K \alpha \beta \phi+r(1-$ $n \Delta t)] / 2 r(\alpha \beta)^{2} \phi \Delta t \quad$ if $\quad K \alpha \beta \phi>r(1-n \Delta t) /(1+r \Delta t)$. We are now ready to detect conditions under which those critical points in $\mathbb{R}$ are mapped into $\mathbb{R}$.

(1) Suppose $K \alpha \beta \phi \leq r(1-n \Delta t) /(1+r \Delta t)$. Consider point $A=\left(S_{A}, 0\right)$ in Fig. A1(A) It is mapped to point $A^{\prime}=$ $\left(S_{A}^{\prime}, 0\right)$ on the horizontal axis where $S_{A^{\prime}}=(1+r \Delta t / 4) S_{0}$. Hence $0 \leq S_{\mathrm{A}^{\prime}} \leq S_{0}$ hold if $0 \leq(1+r \Delta t / 4) \leq 1$, whence $0 \leq S_{\mathrm{A}^{\prime}} \leq S_{0}$ if $0 \leq r \Delta t \leq 3$. Consider point $B=\left(0, L_{0}\right)$. It is mapped to point $B^{\prime}=\left(0, L_{B}^{\prime}\right)$ on the vertical axis where $L_{B}^{\prime}=(1-n \Delta t) L_{0}$. Hence $0 \leq L_{B}^{\prime} \leq L_{0}$ since $0 \leq$ $(1-n \Delta t) \leq 1$ by $n \Delta t \geq 0$ and Assumption. 

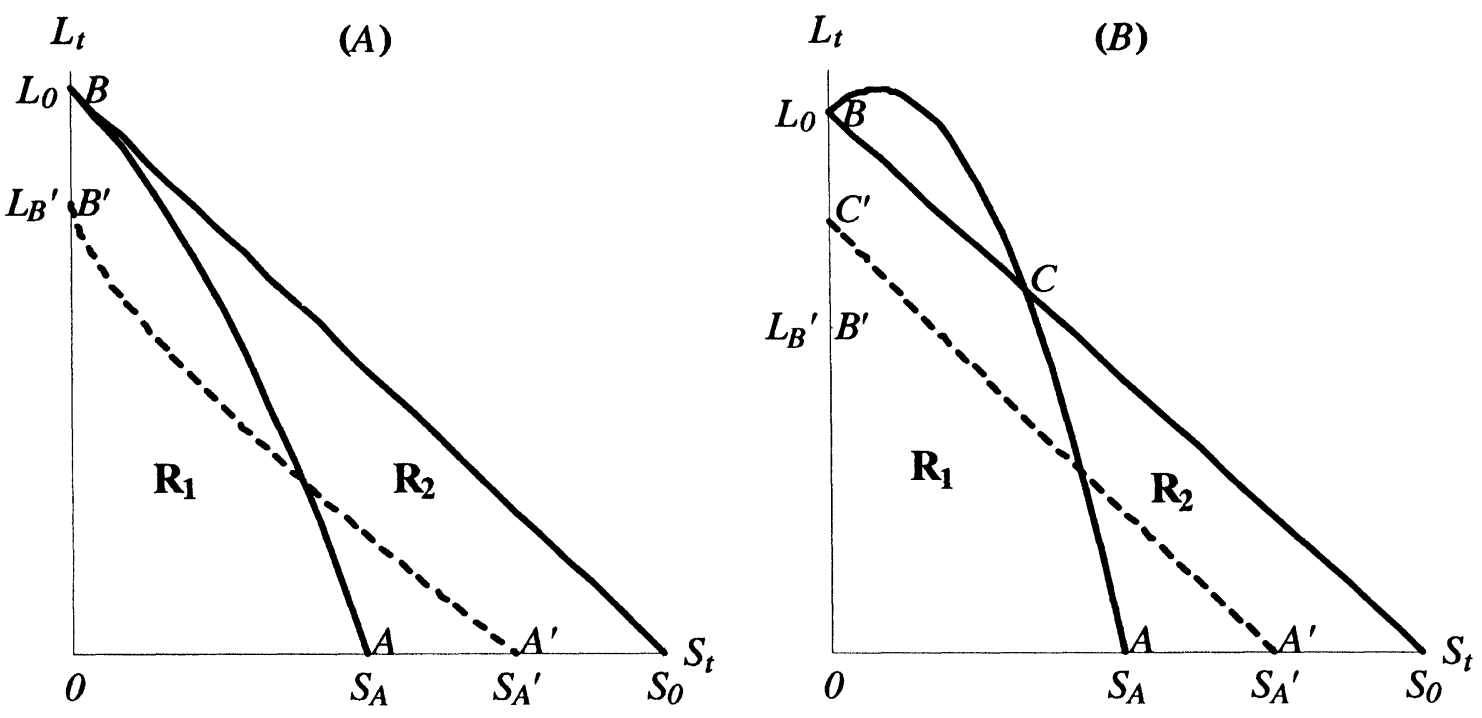

FIGURE A1 Transformation of $\mathbb{R}$. (A) $K \alpha \beta \phi \leq r(1-n \Delta t) /(1+r \Delta t)$; (B) $K \alpha \beta \phi>r(1-n \Delta t) /(1+r \Delta t)$.

(2) Suppose $K \alpha \beta \phi>r(1-n \Delta t) /(1+r \Delta t)$. By considering points $A$ and $B$ in Fig. A1(B), we can also find that $0 \leq S_{A^{\prime}} \leq S_{0}$ if $0 \leq r \Delta t \leq 3$ and $0 \leq L_{B}^{\prime} \leq L_{0}$ if $0 \leq$ $n \Delta t \leq 1$. Point $C$ is an intersection of $f(S)$ and $g(S)$. It is mapped to point $C^{\prime}=\left(0, L_{C}^{\prime}\right)$ on the vertical axis where $L_{C}^{\prime}=\{(1+r \Delta t) K \alpha \beta \phi+r(1-n \Delta t)\}^{2} / 4 r K(\alpha \beta)^{2} \phi \Delta t$. It can be shown that $L_{C}^{\prime} \leq L_{0}$ if $K \alpha \beta \phi \leq r(1+\sqrt{n \Delta t})^{2} / 1+$ $r \Delta t$.

In either of Fig. A1(A) or (B), the real line $A B$ is mapped to the dotted line $A^{\prime} B^{\prime}$, and either of the sub-regions, $\mathbb{R}_{1}$ or $\mathbb{R}_{2}$, is mapped into the triangle $O A^{\prime} B^{\prime}$. Thus the followings are the conditions under which $O A^{\prime} B^{\prime} \subset \mathbb{R}$, namely, $F(\mathbb{R}) \subset \mathbb{R}$.

(1) Suppose $K \alpha \beta \phi \leq r(1-n \Delta t) /(1+r \Delta t)$, then $F(\mathbb{R}) \subset \mathbb{R}$ if $0 \leq n \Delta t \leq 1$ and $0 \leq r \Delta t \leq 3$.

(2) Suppose $K \alpha \beta \phi>r(1-n \Delta t) /(1+r \Delta t)$, then $F(\mathbb{R}) \subset \mathbb{R}$ if $0 \leq n \Delta t \leq 1, \quad 0 \leq r \Delta t \leq 3 \quad$ and $K \alpha \beta \phi \leq r\left(1+\sqrt{n \Delta t)^{2}} /(1+r \Delta t)\right.$

Putting (1) and (2) together, we have proved Theorem 2. 


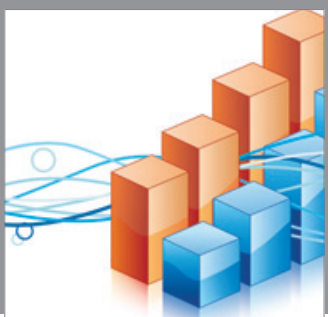

Advances in

Operations Research

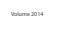

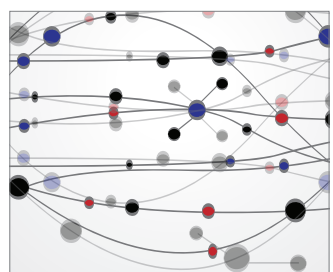

\section{The Scientific} World Journal
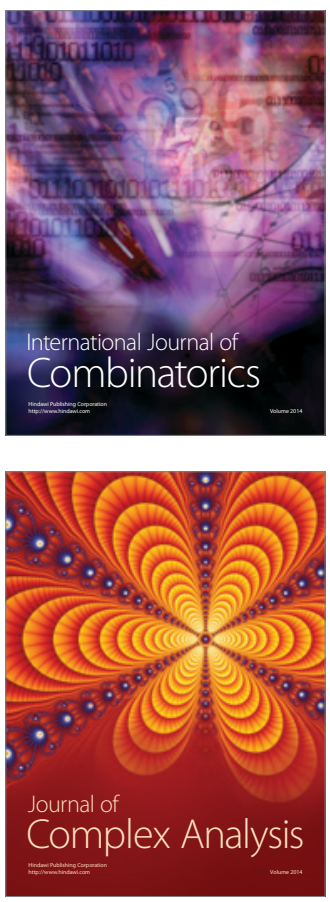

International Journal of

Mathematics and

Mathematical

Sciences
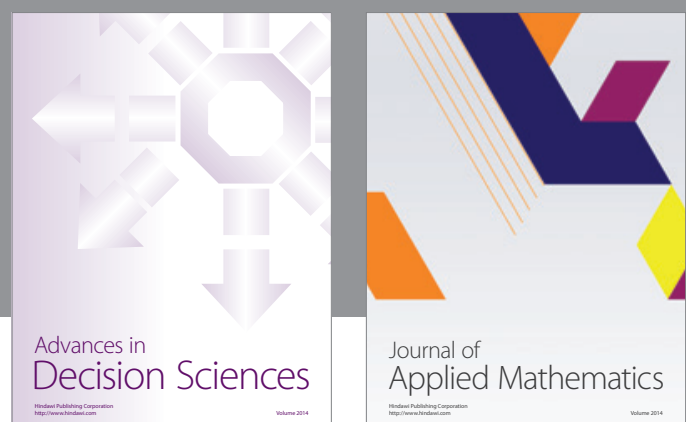

Journal of

Applied Mathematics
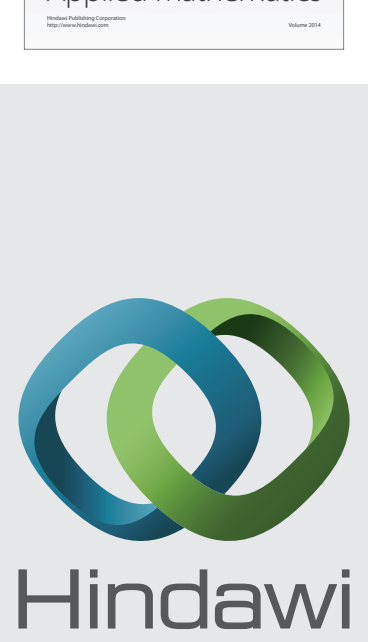

Submit your manuscripts at http://www.hindawi.com
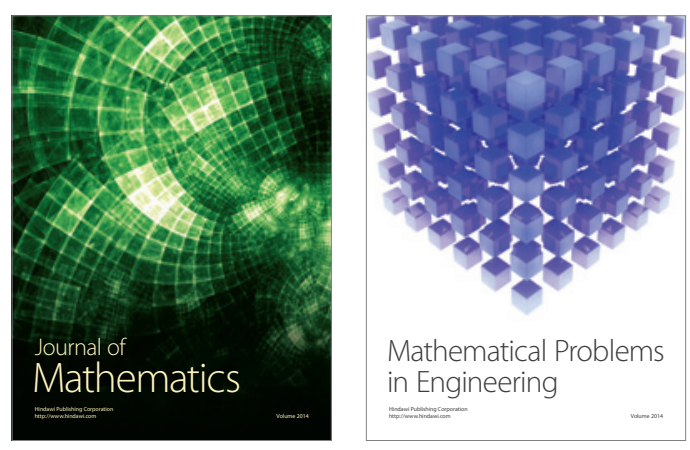

Mathematical Problems in Engineering
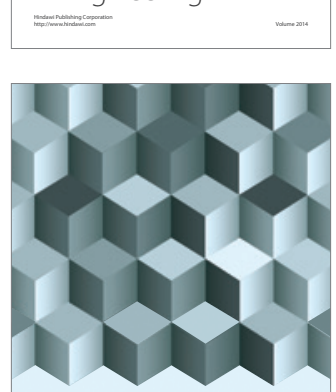

Journal of

Function Spaces
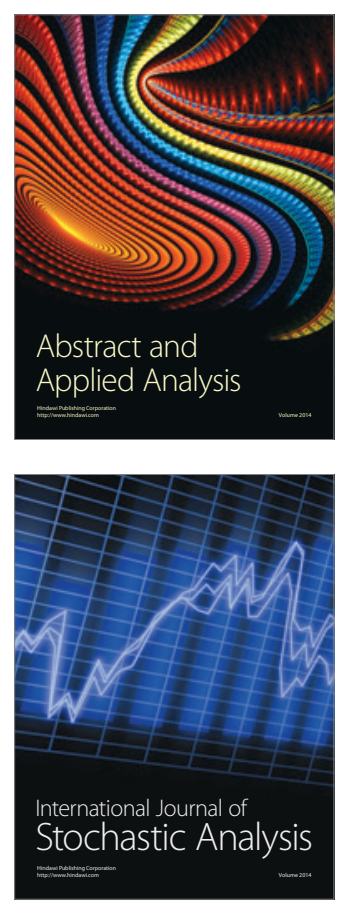

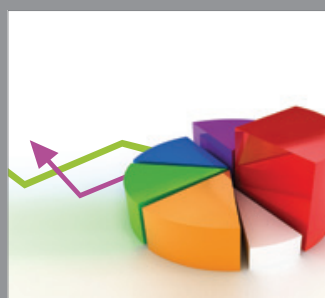

ournal of

Probability and Statistics

Promensencen
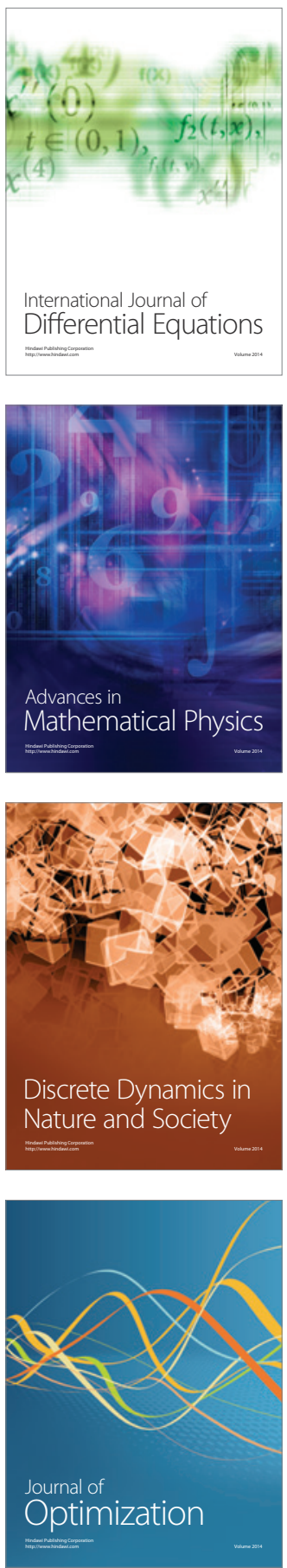